\title{
A JOINT MODEL OF X-RAY AND INFRARED BACKGROUNDS. II. COMPTON-THICK ACTIVE GALACTIC NUCLEUS ABUNDANCE*
}

\author{
Yong Shi ${ }^{1,2,3}$, George Helou ${ }^{1}$, and Lee Armus ${ }^{1}$ \\ ${ }^{1}$ Infrared Processing and Analysis Center, California Institute of Technology, 1200 E. California Boulevard, Pasadena, CA 91125, USA \\ ${ }^{2}$ School of Astronomy and Space Science, Nanjing University, Nanjing 210093, China \\ ${ }^{3}$ Key Laboratory of Modern Astronomy and Astrophysics (Nanjing University), Ministry of Education, Nanjing 210093, China \\ Received 2013 January 23; accepted 2013 July 20; published 2013 October 8
}

\begin{abstract}
We estimate the abundance of Compton-thick (CT) active galactic nuclei (AGNs) based on our joint model of $\mathrm{X}$-ray and infrared backgrounds. At $L_{\mathrm{rest} 2-10 \mathrm{keV}}>10^{42} \mathrm{erg} \mathrm{s}^{-1}$, the CT AGN density predicted by our model is a few $\times 10^{-4} \mathrm{Mpc}^{-3}$ from $z=0$ up to $z=3$. CT AGNs with higher luminosity cuts $\left(>10^{43}, 10^{44}\right.$, and $10^{45} \mathrm{erg} \mathrm{s}^{-1}$ ) peak at higher redshift and show a rapid increase in number density from $z=0$ to $z \sim 2-3$. The CT AGN to all AGN ratio appears to be low (2\%-5\%) at $f_{2-10 \mathrm{keV}}>10^{-15} \mathrm{erg} \mathrm{s}^{-1} \mathrm{~cm}^{-2}$ but rises rapidly toward fainter flux levels. The CT AGNs account for $\sim 38 \%$ of the total accreted supermassive black hole mass and contribute $\sim 25 \%$ of the cosmic X-ray background spectrum at $20 \mathrm{keV}$. Our model predicts that the majority (90\%) of luminous and bright CT AGNs $\left(L_{\mathrm{rest} 2-10 \mathrm{keV}}>10^{44} \mathrm{erg} \mathrm{s}^{-1}\right.$ or $\left.f_{2-10 \mathrm{keV}}>10^{-15} \mathrm{erg} \mathrm{s}^{-1} \mathrm{~cm}^{-2}\right)$ have detectable hot dust 5-10 $\mu \mathrm{m}$ emission, which we associate with a dusty torus. The fraction drops for fainter objects, to around $30 \%$ at $L_{\text {rest2-10 keV }}>10^{42} \mathrm{erg} \mathrm{s}^{-1}$ or $f_{2-10 \mathrm{keV}}>10^{-17} \mathrm{erg} \mathrm{s}^{-1} \mathrm{~cm}^{-2}$. Our model confirms that heavily obscured AGNs $\left(N_{\mathrm{HI}}>10^{23} \mathrm{~cm}^{-2}\right)$ can be separated from unobscured and mildly obscured ones $\left(N_{\mathrm{HI}}<10^{23} \mathrm{~cm}^{-2}\right)$ in the plane of observed frame X-ray hardness versus mid-IR/X-ray ratio.
\end{abstract}

Key words: galaxies: active - galaxies: nuclei

Online-only material: color figures

\section{INTRODUCTION}

Active galactic nuclei (AGNs) with Compton-thick (CT) nuclear obscuration $\left(N_{\mathrm{HI}}>1.5 \times 10^{24} \mathrm{~cm}^{-2}\right)$ are crucial in the quest for a complete census of the AGN population. While Chandra and XMM-Newton have revealed a large population of AGNs, up to $z \sim 5$, and demonstrated unambiguously the dominance of supermassive black hole (SMBH) accretion in the obscured phase (Mainieri et al. 2002; Perola et al. 2004; Hasinger et al. 2005; Barger et al. 2005), the necessity of having a high signal-to-noise X-ray spectrum, including detection above and below rest-frame energies of $10 \mathrm{keV}$, largely limits the ability to detect the presence of CT AGNs in deep surveys (Tozzi et al. 2006; Georgantopoulos et al. 2009). However, there are several compelling reasons to suspect an abundant distant CT AGN population: (1) in the local universe the CT AGNs comprise roughly $50 \%$ of the optically selected AGN sample (Risaliti et al. 1999; Guainazzi et al. 2005); given the dusty high- $z$ universe, the distant CT AGNs may be more abundant. (2) Cosmic X-ray background (CXB) population models invoke luminosity functions (LFs) of AGNs with different $\mathrm{H}$ I columns to fit the X-ray survey data (Comastri et al. 1995; Gilli et al. 2007; Treister et al. 2009b), which requires a large number of CT AGNs to reproduce the CXB spectrum at its peak (20-30 keV); this general conclusion is largely independent of detailed assumptions in the model. (3) The multi-wavelength techniques that combine the X-ray data with optical/IR photometry offer powerful ways to identify CT candidates, and indicate an increasing spatial density of CT AGNs with redshift (Alexander et al. 2008; Daddi et al. 2007; Fiore et al. 2009; Luo et al. 2011; Treister et al. 2009a).

\footnotetext{
* Online calculators of the model are available at

http://5muses.ipac.caltech.edu/5muses/EBL_model/index.html.
}

In Shi et al. (2013, hereafter Paper I), we presented a joint population model of X-ray and IR backgrounds that fits the survey data in the $0.5-60 \mathrm{keV}$ and $24-1200 \mu \mathrm{m}$ bands, with the goal of studying the cosmic evolution of AGNs and dusty starbursts. We discuss here the CT AGN abundance derived from this model. In contrast to CXB models, which only fit $\mathrm{X}$-ray data primarily at energies below $10 \mathrm{keV}$, our approach is fundamentally different. CXB models usually use $0.1-10 \mathrm{keV}$ data to fit the Compton-thin AGN counts, extrapolate the result to $20-30 \mathrm{keV}$, and then subtract this from the CXB spectrum to derive the abundance of CT AGNs. Our model constrains the Compton-thin AGNs from 0.5-10 keV data and starburst galaxies from far-IR data, and compares the $24 \mu \mathrm{m}$ from these populations to the IR background and known distributions of $24 \mu \mathrm{m}$-detected sources. The residual of the $24 \mu \mathrm{m}$ emission, after subtracting the contributions from Compton-thin and starburst galaxies, is assumed to be from CT AGNs. As a result, our model uses more observational information to constrain the CT AGN fractions as a function of luminosity and redshift, including both number counts and redshift distributions at $24 \mu \mathrm{m}$. In our model, we allow the redshift evolution of the CT AGN to be a free parameter, while the CXB models typically assume no evolution, fixed evolution, or an evolution that is the same as the Compton-thin AGN.

The spectral energy distribution (SED) of individual sources is crucial to any population model, but our joint model and the CXB models depend on different parts of the SED. The CXB model is sensitive to the X-ray SED above $10 \mathrm{keV}$ (e.g., Gilli et al. 2007; Treister et al. 2009b), while ours relies on the $24 \mu \mathrm{m} / 2-10 \mathrm{keV}$ ratio of the SMBH SED but also the starforming IR SED. As a result, we ran four variants of the model to incorporate the SED uncertainties, including the reference one, the one with X-ray to IR ratio of the SMBH SED $3 \sigma$ ( $0.2 \mathrm{dex})$ above the average ratio, the one with X-ray to IR ratio 


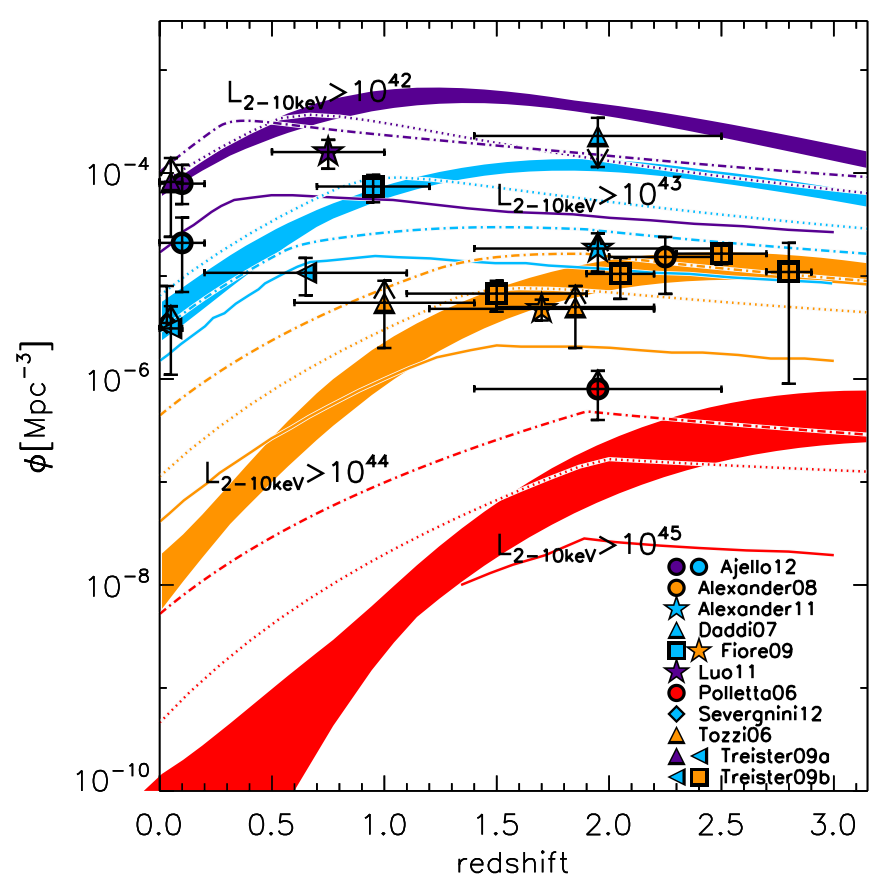

Figure 1. Comoving number density of CT AGNs above different intrinsic $2-10 \mathrm{keV}$ luminosities as a function of redshift. The filled areas represent the predictions of our model in each of the four luminosity bins. The width of the curves reflects the uncertainties of the predictions. The solid, dotted, and dot-dashed lines are the predictions by the CXB models of Treister et al. (2009b), Gilli et al. (2007), and Draper \& Ballantyne (2010), respectively. All symbols represent the observations. A symbol is plotted with a filled core plus a black envelope. For all filled areas, lines, and symbol cores, purple: $L_{2-10 \mathrm{keV}}>10^{42} \mathrm{erg} \mathrm{s}^{-1}$, cyan: $L_{2-10 \mathrm{keV}}>10^{43} \mathrm{erg} \mathrm{s}^{-1}$, orange: $L_{2-10 \mathrm{keV}}>10^{44} \mathrm{erg} \mathrm{s}^{-1}$, and red: $L_{2-10 \mathrm{keV}}>10^{45} \mathrm{erg} \mathrm{s}^{-1}$

(A color version of this figure is available in the online journal.)

of the SMBH SED $3 \sigma(0.2$ dex $)$ below the average ratio, and the one assuming strong redshift evolution in the starforming SED (for details, see Paper I). Overall, our model provides a new way to constrain the CT AGN abundance using substantially more information from more diverse deep survey data. Paper I presented the detailed model construction and three basic outputs including the total IR LF, the SMBH energy fraction in the IR band, and H I column density distributions as a function of X-ray luminosity and redshift.

In this paper we discuss the predicted CT AGN abundance and compare it to a large collection of empirical constraints. In Section 2, we show the spatial number density of CT AGNs. We present the type-2 and CT AGN fraction as a function of X-ray fluxes in Section 3. The contribution of CT AGNs to the SMBH accretion and CXB spectrum is shown in Section 4. Discussions and conclusions are presented in Sections 5 and 6, respectively.

\section{THE COMOVING NUMBER DENSITY OF CT AGNs}

Figure 1 shows the predicted comoving number density of CT AGNs above different intrinsic rest-frame $2-10 \mathrm{keV}$ luminosity limits as a function of redshift (filled areas with different colors). Symbols with the same color are the empirical estimates from the literature above the same limits. Among the different variants of our model, as reflected by the vertical width of the filled area in the figure, the predicted number density varies from a factor of two for low luminosity objects $\left(>10^{42} \mathrm{erg} \mathrm{s}^{-1}\right)$ up to a factor of five for luminous ones $\left(>10^{45} \mathrm{erg} \mathrm{s}^{-1}\right)$. For CT AGNs with intrinsic $L_{\text {rest2-10 }} \mathrm{keV}>10^{42} \mathrm{erg} \mathrm{s}^{-1}$, our model predicts the density will peak at a few $\times 10^{-4} \mathrm{Mpc}^{-3}$ around $z \sim 1-1.5$, declining slowly toward both higher and lower redshifts. The local densities, as measured by Treister et al. (2009b) and Ajello et al. (2012), are consistent with our predictions. The estimate at $z \sim 0.7$ by Luo et al. (2011) is only a factor of two lower than our prediction. They performed a Monte Carlo simulation to estimate the CT AGN fraction in a sample of IR-excess sources defined as having excess IR emission relative to the UV-based star-forming emission $\left(\log \left(\mathrm{SFR}_{\mathrm{IR}+\mathrm{UV}} / \mathrm{SFR}_{\mathrm{UV} \text {, corr }}\right)>0.5\right.$; e.g., Daddi et al. 2007). The stacked X-ray spectrum of these sources shows evidence of heavy extinction. However, due to the lack of CT signatures in individual galaxies, this statistical approach still shows large uncertainties.

The more luminous CT AGNs at $L_{2-10 \mathrm{kev}}>10^{43} \mathrm{erg} \mathrm{s}^{-1}$ show a different trend, with a faster evolution starting at $z=0$ and a higher peak redshift. The predicted local density is consistent with the empirical estimate by Treister et al. (2009b) and Severgnini et al. (2012) but three times lower than that by Ajello et al. (2012), yet still within the uncertainty. The work by Treister et al. (2009b) only includes the transmission AGNs, thus underestimating the total population if reflectiondominated CT AGNs are abundant. Beyond the local universe, the density around $z=0.7$ by Treister et al. (2009a) is three times lower than the prediction of our model, while other high- $z$ studies by Fiore et al. (2009), Daddi et al. (2007), and Alexander et al. (2011) give results consistent with the model. Among them, Daddi et al. (2007) give a solid upper limit, as they assume a CT nature for all of their IR-excess objects $\left(\log \left(\mathrm{SFR}_{\mathrm{IR}+\mathrm{UV}} / \mathrm{SFR}_{\mathrm{UV} \text {, corr }}\right)>0.5\right)$, while Alexander et al. (2011) derived a solid lower limit by only counting sources that satisfy the $B z K$ selection detected in the X-ray.

At $L_{2-10 \mathrm{keV}}>10^{44} \mathrm{erg} \mathrm{s}^{-1}$, the comoving density of the CT AGN shows a rapid rise until $z \sim 2$ and almost a flat trend up to $z=3$. The model's prediction is more or less consistent with previous observations (Alexander et al. 2008; Fiore et al. 2009; Treister et al. 2009a), except for one data point around $z \approx 0.9$, but within the uncertainty (Tozzi et al. 2006). Tozzi et al. (2006) analyzed the X-ray spectra of sources in the $1 \mathrm{Ms}$ CDF-S and identified $14 \mathrm{CT}$ AGNs, possibly missing the X-ray-undetected CT AGNs. At $L_{2-10 \mathrm{keV}}>10^{45} \mathrm{erg} \mathrm{s}^{-1}$, the CT AGN shows a rapid evolution in the model, with two orders of magnitude increase in density from $z=0$ up to $z=3$. The model prediction around $z \sim 2$ is lower than the estimate by Polletta et al. (2006). Although only X-ray-detected sources are accounted for in this measurement (Treister et al. 2009b), the low number of objects potentially indicates a large uncertainty associated with the measurement.

The predicted CT AGN number density of our model is generally similar to predictions by the CXB model of Gilli et al. (2007; dotted lines in Figure 1), but ours peaks at higher redshift. The model of Gilli et al. (2007) assumed all X-ray sources detected at $0.5-2$ and $2-10 \mathrm{keV}$ to be Compton-thin and derived their distributions as a function of redshift, luminosity, and $\mathrm{H}$ I columns. After subtracting the contribution of these Comptonthin sources from the CXB spectrum, the residual is not zero, thus implying the existence of CT AGNs. By assuming the same redshift evolution for CT AGNs as for obscured Comptonthin AGNs, the number of CT AGNs is derived by matching to the CXB spectrum. At a given redshift and limiting X-ray luminosity, their results do not significantly deviate from ours. However, a significant difference between the two models is that they predict a lower redshift for the peak of the CT AGN number density. For $L_{2-10 \mathrm{keV}}>10^{42} \mathrm{erg} \mathrm{s}^{-1}$, our peak redshift is $z \sim$ $1-1.5$ compared to theirs, which is around $z \sim 0.7$. A difference 

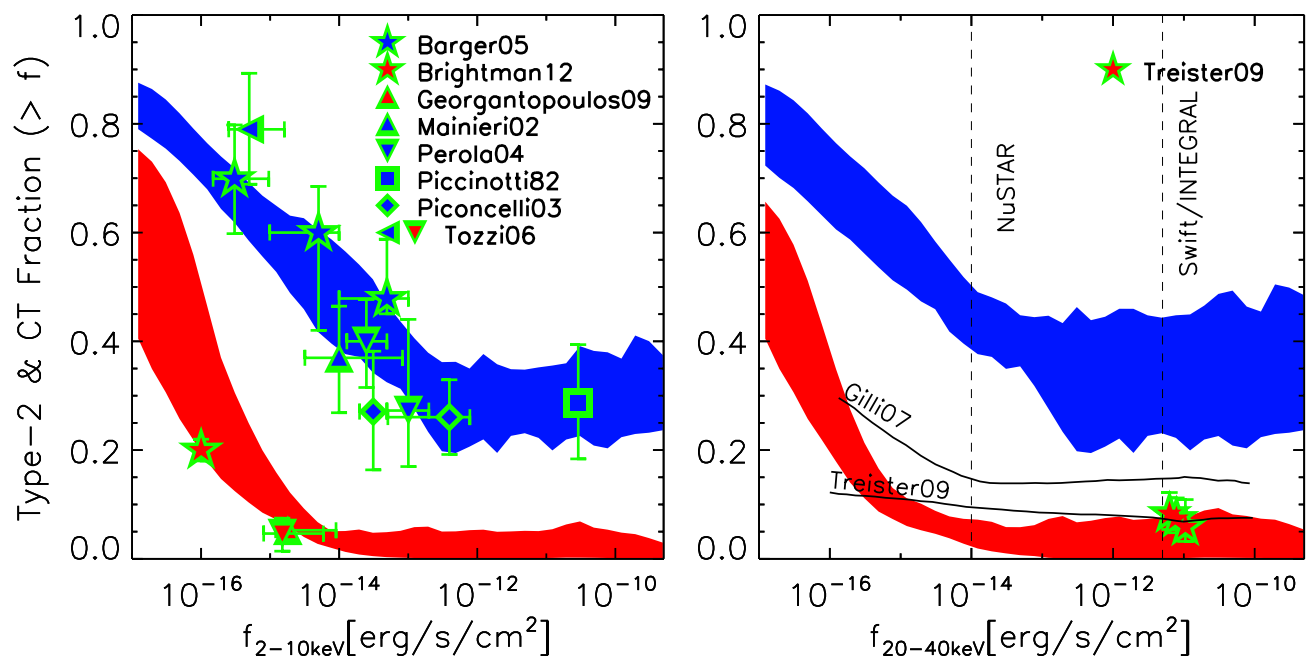

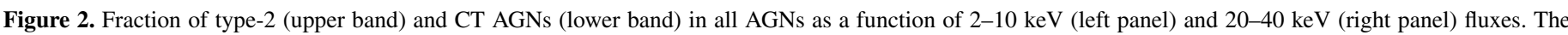

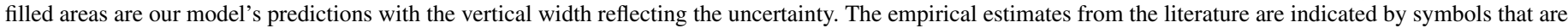

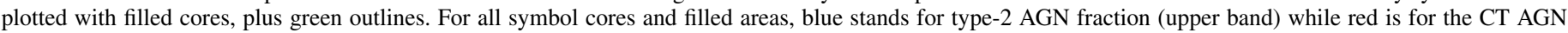

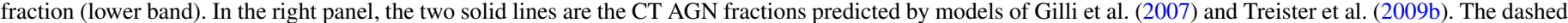
lines give the typical survey limits of NUSTAR, Swift, and INTEGRAL.

(A color version of this figure is available in the online journal.)

of $\Delta z=0.5-1$ in the peak redshift is also found for the number density of CT AGNs at $L_{2-10 \mathrm{keV}}>10^{43,44,45} \mathrm{erg} \mathrm{s}^{-1}$.

The CXB model of Treister et al. (2009b; solid lines in Figure 1) predicts lower CT AGN number densities than ours. At $L_{2-10 \mathrm{keV}}>10^{42} \mathrm{erg} \mathrm{s}^{-1}$, our predicted CT density is 5-10 times higher than theirs across the whole redshift range. At higher luminosities $\left(>10^{43,44,45} \mathrm{erg} \mathrm{s}^{-1}\right.$ ), their predictions are similar to ours up to their turn-over redshift, but lower by 5-10 at higher redshifts as our predicted density rises faster. This is not surprising, since Treister et al. hold the CT fraction in obscured AGNs constant at the local value, seen in the INTEGRAL and Swift data, allowing for no redshift evolution. In contrast, our model requires more $\mathrm{CT}$ AGNs at higher redshifts to satisfy the various sets of observations (see Paper I). As shown in the next section, our model has no problem in reproducing the CT AGN fraction as observed by INTEGRAL and Swift.

In the CXB model of Draper \& Ballantyne (2010; dot-dashed lines in Figure 1), low-luminosity CT AGNs $\left(>10^{42} \mathrm{erg} \mathrm{s}^{-1}\right.$ and $>10^{43}$ ) have comparable number densities to those predicted from our models at low redshift, but are noticeably lower than ours at high redshift. The opposite seems to be the case for high-luminosity CT AGNs, where the two models make similar predictions at high redshift, but differ at low redshift where the Draper \& Ballantyne (2010) models predict more CT AGNs than our models. These differences reflect the weaker dependence of their models on luminosity and redshift (Draper \& Ballantyne 2009, 2010).

\section{THE TYPE-2 AND CT AGN FRACTION}

Figure 2 plots the fractions of AGN that are type-2 $\left(N_{\mathrm{H}_{\mathrm{I}}}>\right.$ $10^{22} \mathrm{~cm}^{-2}$ ) and CT at different $2-10 \mathrm{keV}$ and $20-40 \mathrm{keV}$ fluxes. The two fractions show similar behaviors, i.e., a flat trend at bright fluxes along with a rapid rise toward fainter ends. Constant type- 2 fractions of $30 \% \pm 10 \%$ and $35 \% \pm 10 \%$ are found at $2-10 \mathrm{keV}$ and $20-40 \mathrm{keV}$ above fluxes of $10^{-13} \mathrm{erg} \mathrm{s}^{-1} \mathrm{~cm}^{-2}$, respectively, while the $\mathrm{CT}$ AGN fractions remain around $3 \% \pm$ $3 \%$ and $4 \% \pm 3 \%$ at $2-10 \mathrm{keV}$ and $20-40 \mathrm{keV}$ above fluxes of
$10^{-15} \mathrm{erg} \mathrm{s}^{-1} \mathrm{~cm}^{-2}$, respectively. In Paper I, our model predicts a rapid redshift evolution of the type- 2 and $\mathrm{CT}$ AGN fractions at given intrinsic $\mathrm{X}$-ray luminosities, causing the two fractions to increase with decreasing flux which, combined with further obscuration to $\mathrm{CT} /$ type- 2 objects, results in a flat trend at bright fluxes but a rapid rise toward lower fluxes.

As shown in the figure, the predicted type-2 AGN fractions are consistent with empirical constraints as compiled in the work of Gilli et al. (2007) as well as Barger et al. (2005), Mainieri et al. (2002), Perola et al. (2004), Piccinotti et al. (1982), Piconcelli et al. (2003), and Tozzi et al. (2006). All of these studies identified the type- 2 in X-ray flux-limited samples through X-ray spectral analysis. For the CT AGN fraction as a function of the $2-10 \mathrm{keV}$ flux, we compared our predictions to empirical estimates based on studies of three X-ray fluxlimited samples (Tozzi et al. 2006; Georgantopoulos et al. 2009; Brightman \& Ueda 2012) that are constructed from Chandra $1 \mathrm{Ms}, 2 \mathrm{Ms}$, and $4 \mathrm{Ms}$ survey data, respectively. The first two identified CT AGNs through X-ray spectral analysis and derived CT fractions of $5 \%$ down to $f_{2-10 \mathrm{keV}}=10^{-15} \mathrm{erg} \mathrm{s}^{-1} \mathrm{~cm}^{-2}$, consistent with the predictions of our model. The majority of these objects are CT AGNs whose transmitted light dominates over the reflected radiation in X-ray. Brightman \& Ueda (2012) carried out X-ray spectral analysis of 449 X-ray sources down to a flux 10 times lower $\left(f_{2-10 \mathrm{keV}}=10^{-16} \mathrm{erg} \mathrm{s}^{-1} \mathrm{~cm}^{-2}\right)$, with average photon counts 3-5 times smaller than the other two studies. After corrections for these two effects, they argued for $20 \% \pm 2 \%$ CT AGN fraction, lower than our model's prediction but within the uncertainty. At $20-40 \mathrm{keV}$, our result is consistent with the CT fraction as identified in Swift/INTEGRAL data (Treister et al. 2009b), in which all CT AGNs are identified as transmission sources. The recently launched NUSTAR mission should offer strong constraints on the CT AGN fraction down to $10^{-14} \mathrm{erg} \mathrm{s}^{-1} \mathrm{~cm}^{-2}$.

As already discussed in the previous section, our model predicts roughly the same CT AGN abundance as that of Gilli et al. (2007) but our predicted CT AGN number density peaks at higher redshift. This is also reflected in Figure 2 


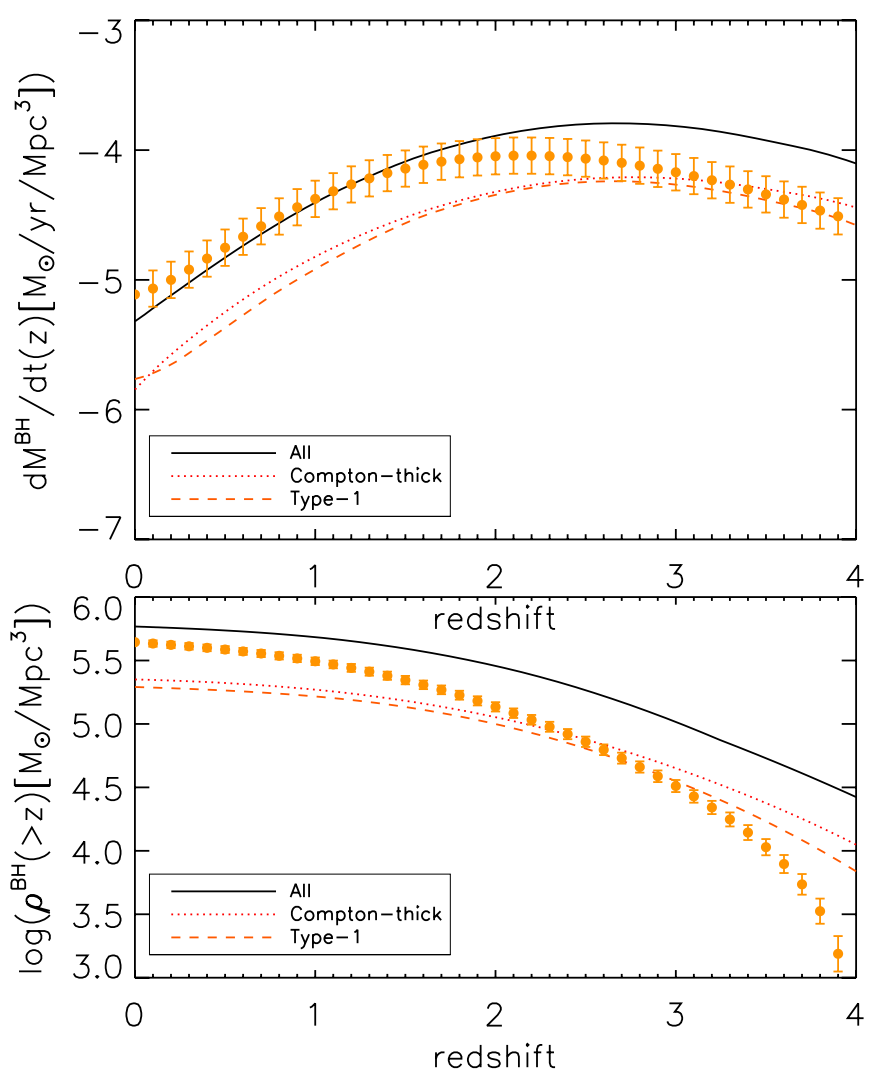

Figure 3. Comoving SMBH accretion rate (upper panel) and total accreted SMBH mass density (lower panel) for all AGNs (solid lines), type-1 (dashed lines), and CT (dotted lines) predicted from our model. Each line is the median value of the predictions from four model variants (see Paper I), while the uncertainty of the predictions among model variants is around $50 \%$. Symbols are from Hopkins et al. (2007). Our predicted accretion rates seem to match those presented in Hopkins et al. (2007) up to $z=2$, but exceed theirs at higher redshifts, while the discrepancy in the cumulative accreted SMBH mass across the redshift is caused by that above $z=2$.

(A color version of this figure is available in the online journal.)

(right panel) where our CT fraction is lower than theirs above $10^{-15} \mathrm{erg} \mathrm{s}^{-1} \mathrm{~cm}^{-2}$ but exceeds theirs at fainter flux levels. Compared to the model of Treister et al. (2009b), our model predicts more abundant CT AGNs at high redshift, resulting in a similar CT fraction above $10^{-15} \mathrm{erg} \mathrm{s}^{-1} \mathrm{~cm}^{-2}$ but a significantly higher fraction at fainter fluxes.

\section{THE CONTRIBUTION OF CT AGNs TO SMBH GROWTH AND CXB}

An SMBH grows through accretion, and the accretion disk is responsible for the optical through X-ray emission. By introducing the radiative efficiency $\epsilon_{r}$, the observed SMBH luminosity and accretion rate are related by:

$$
L_{\mathrm{ox}}^{\prime}=\epsilon_{r} \dot{M}_{\mathrm{BH}} c^{2}
$$

where $L_{\mathrm{ox}}^{\prime}$ is the luminosity integrated from the optical to the Xray $(1 \mu \mathrm{m}$ to $200 \mathrm{keV}), \epsilon_{r}$ is the mass to energy conversion rate, $\dot{M}_{\mathrm{BH}}$ is the $\mathrm{SMBH}$ mass accretion rate, and $c$ is the speed of light. As constructed in Paper I, our quasar SED invokes the luminosity-dependent optical to X-ray ratio, and thus the correction from 2 to $10 \mathrm{keV}$ to the luminosity from $1 \mu \mathrm{m}$ to $200 \mathrm{keV}$ is luminosity dependent with an average value around a factor of 30 . Figure 3 shows the comoving

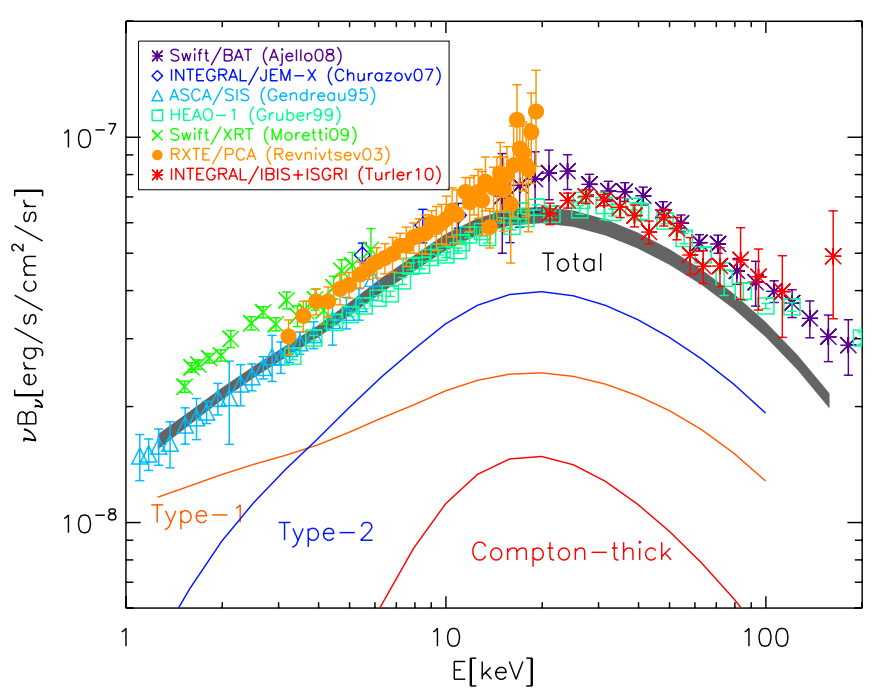

Figure 4. CXB spectrum from our model compared to the observations from the literature (all symbols). The gray curve is the prediction from our model, with the thickness of the curve representing the uncertainty in the model as a function of energy. Colored lines represent the median values of predictions by four model variants (see Paper I) for type-1 (orange), type-2 (blue), and CT (red) AGNs as a function of energy. References: Ajello08: Ajello et al. (2008); Churazov07: Churazov et al. (2007); Gendreau95: Gendreau et al. (1995); Gruber99: Gruber et al. (1999); Moretti09: Moretti et al. (2009); Revnivtsev03: Revnivtsev et al. (2003); Turler10: Türler et al. (2010).

(A color version of this figure is available in the online journal.)

SMBH accretion rate (upper panel) and the cumulative accreted SMBH mass above a given redshift (lower panel). Our predicted accretion rates seem to match those presented in Hopkins et al. (2007) up to $z=2$, but exceed theirs at higher redshifts, while the discrepancy in the cumulative accreted SMBH mass across the redshift is caused by that above $z=2$. We believe the discrepancy to be related to the large uncertainties in deriving the obscured AGN LFs at these redshifts. The derived local SMBH mass density is $(5.8 \pm 1.0) \times 10^{5} M_{\odot} \mathrm{Mpc}^{-3}$ given $\epsilon_{r}=0.1$. This number is consistent with those derived from local bulge mass functions through the bulge/BH-mass relationship, $(2.9 \pm 0.5) \times 10^{5} M_{\odot} \mathrm{Mpc}^{-3}$ (Yu \& Tremaine 2002), (4.2 \pm 1.1$) \times 10^{5} \mathrm{M}_{\odot} \mathrm{Mpc}^{-3}$ (Shankar et al. 2004), and $4.6_{-1.4}^{+1.9} \times 10^{5} M_{\odot} \mathrm{Mpc}^{-3}$ (Marconi et al. 2004). Our model further predicts that only $33 \%$ of local SMBH mass is accreted in the type-1 phase while the $\mathrm{CT}$ accretion contributes as much as $38 \%$ to the local SMBH mass density.

Figure 4 shows our prediction for the CXB spectrum at 1-200 keV as compared with observations. Our model predicts a peak at around $20 \mathrm{keV}$. Below $20 \mathrm{keV}$, our prediction matches the results of RXTE/PCA (Revnivtsev et al. 2003) and $A S C A / S I S$ (Gendreau et al. 1995), but is about 20\% lower than those of Swift/XRT (Moretti et al. 2009) and INTEGRAL/ JEM-X (Churazov et al. 2007). At 20-100 keV, the model is systematically lower by $20 \%-30 \%$ than the observations (Gruber et al. 1999; Ajello et al. 2008; Türler et al. 2010). We do not know exactly what causes the discrepancy but we have noticed that the model cannot reproduce the local $15-55 \mathrm{keV}$ counts of Ajello et al. (2012) and the CXB spectrum above $20 \mathrm{keV}$ at the same time. If the model fits the Ajello et al. (2012) counts, it underproduces the CXB spectrum above $20 \mathrm{keV}$. However, if the model is forced to fit the $\mathrm{CXB}$ spectrum, it would overpredict the counts of Ajello et al. (2012). As noted in Ajello et al. (2012), previous CXB models have a similar problem, 
including those of Gilli et al. (2007), Treister et al. (2009b), and Draper \& Ballantyne (2010), where they fit the CXB spectrum well but overpredict the local $15-55 \mathrm{keV}$ counts. A key observational input for the CXB models to fit both the Ajello et al. (2008) counts and the CXB spectrum above $20 \mathrm{keV}$ is the rest-frame SED at energies above $20 \mathrm{keV}$, which is still not well constrained given limited observations. But we cannot exclude the possibility that our model has limitations in reproducing the CXB spectrum above $20 \mathrm{keV}$, as it fits so many data points over a very large frequency range (X-ray and $\mathrm{IR} /$ submm) to minimize $\chi^{2}$.

As shown in Figure 4, type-1 AGNs dominate the CXB below $5 \mathrm{keV}$, above which type-2s are responsible for the majority of CXB emissions. The CT AGN contribution rises quickly from low energy and peaks around $\sim 25 \%$ at $20 \mathrm{keV}$.

\section{DISCUSSION}

Our model predicts an abundant CT AGN population, especially at high- $z$. By comparing Figure 1 to the type-1 unobscured AGN number density as measured by Hasinger et al. (2005), our predicted CT AGN density is 3-5 times higher at $L_{2-10 \mathrm{keV}}>10^{42} \mathrm{erg} \mathrm{s}^{-1}$. At $L_{2-10 \mathrm{keV}}>10^{44} \mathrm{erg} \mathrm{s}^{-1}$, the predicted CT AGN density is still comparable to their type-1 AGN, around $z \sim 2$. As shown in Paper I, our model predicts an increasing CT AGN fraction with redshift, resulting in a larger CT AGN population at high redshift as compared to the predictions of Gilli et al. (2007) and Treister et al. (2009b). The large CT AGN fraction around $z \sim 2$ may be consistent with observational evidence for high gas fractions and associated high star formation rates (SFRs) of $z \sim 2$ galaxies (Tacconi et al. 2010). The high velocity dispersion of $z \sim 2$ gas disks implies a large vertical height (Förster Schreiber et al. 2006; Law et al. 2007; Genzel et al. 2008), as might result from continuous stirring by ongoing star formation (Elmegreen \& Burkert 2010). If such behavior persists as gas is transported down to the central 1-10 pc scale around the nuclear BH (Wada \& Norman 2002; Hopkins et al. 2012), the dusty torus of high redshift AGNs might have a larger vertical extent and subsequently cover a larger solid angle, resulting in a larger CT AGN fraction at high redshift (Fabian 1999). Despite their abundance in our model, as shown in Figure 2, CT AGNs only dominate at $f_{2-10 \mathrm{keV}}<10^{-15} \mathrm{erg} \mathrm{s}^{-1} \mathrm{~cm}^{-2}$, where few data are available from current X-ray missions to reliably identify these objects. Another possibility to explain the increasing obscured fraction is related to redshift evolution of major mergers, as shown by Treister et al. (2010), in which abundant dust and gas brought in by mergers obscure the nucleus before they are disrupted by radiation pressure to reveal a type-1, unobscured quasar phase.

The featureless mid-IR emission from the dusty torus of AGNs has been a powerful method to infer the intrinsic accretion luminosity. In particular, when appearing along with weak or no X-ray emission, this continuum offers a strong indicator of CT H i columns (Lacy et al. 2004; Stern et al. 2005; AlonsoHerrero et al. 2006; Treister et al. 2009a; Alexander et al. 2008). Figure 5 gives the predicted median and 20\%-80\% probability range of $6.2 \mu \mathrm{m}$ aromatic feature EW of CT AGNs in three intrinsic X-ray luminosity ranges, $10^{42}-10^{43} \mathrm{erg} \mathrm{s}^{-1}$, $10^{43}-10^{44} \mathrm{erg} \mathrm{s}^{-1}$, and $>10^{44} \mathrm{erg} \mathrm{s}^{-1}$. Note that the EW of star-forming templates in our model has a value of $0.6 \mu \mathrm{m}$, so smaller values indicate the presence of the contribution from the dusty torus. In Paper I, we compared our model's predictions on the EW distributions to observations for two Spitzer legacy programs (GOALS and 5MUSES) and found a

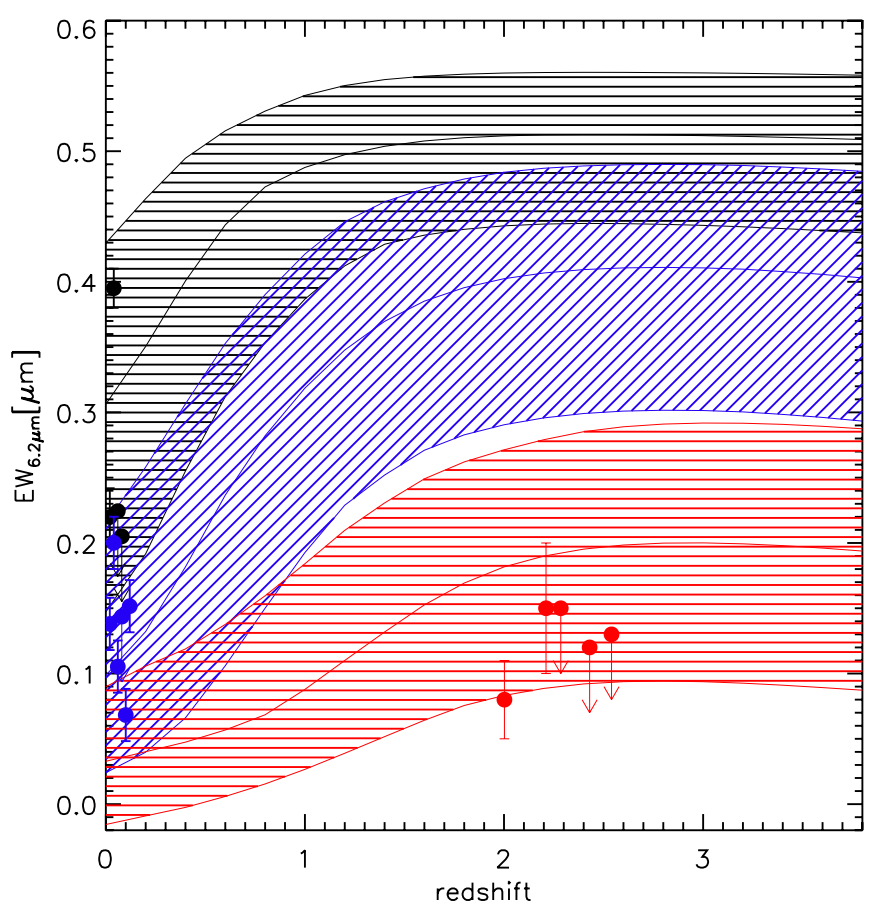

Figure 5. Predicted median $\mathrm{EW}_{6.2} \mu \mathrm{mPAH}$ and $20 \%-80 \%$ probability range of CT AGNs in three intrinsic X-ray luminosity ranges. Symbols are observations of low- $z$ (Risaliti et al. 1999) and high-z (Alexander et al. 2008) CT AGNs. For all shaded areas and symbols, black: $L_{2-10 \mathrm{keV}}=10^{42}-10^{43} \mathrm{erg} \mathrm{s}^{-1}$, blue: $L_{2-10 \mathrm{keV}}=10^{43}-10^{44} \mathrm{erg} \mathrm{s}^{-1}$, and red: $L_{2-10 \mathrm{keV}}>10^{44} \mathrm{erg} \mathrm{s}^{-1}$. Note that in our model, pure star formation has an EW of $0.6 \mu \mathrm{m}$; any value below that is due to contributions from AGN emission.

(A color version of this figure is available in the online journal.)

general consistency. Figure 5 also gives the observed EW of individual CT AGNs drawn from the literature, where the local sample is from Risaliti et al. (1999), and the high- $z$ sample is from Alexander et al. (2008). Although a fair comparison between the model and the observation is impossible for such a sample, the data are within the model's predicted range. Figure 6 further shows the $6.2 \mu \mathrm{m}$ feature EW distribution of AGNs above different intrinsic X-ray luminosities. We define objects with $\mathrm{EW}_{6.2 \mu \mathrm{m}}<0.4 \mu \mathrm{m}$ as those with detectable hot dust emission from AGNs, because observations confirm a small scatter in the star-forming $6.2 \mu \mathrm{m}$ EWs, with the median of $0.6 \mu \mathrm{m}$ and $3 \sigma$ dispersions about $0.2-0.25 \mu \mathrm{m}$ (e.g., $\mathrm{Wu}$ et al. 2010; Stierwalt et al. 2013). The figure indicates that the fractions of CT AGNs that have $\mathrm{EW}_{6.2 \mu \mathrm{m}}<0.4$, i.e., detectable AGN mid-IR emission, are $27 \%, 55 \%, 98 \%$, and $100 \%$ for the limiting intrinsic rest-frame $2-10 \mathrm{keV}$ luminosities of $10^{42}, 10^{43}$, $10^{44}$, and $10^{45} \mathrm{erg} \mathrm{s}^{-1}$, respectively. Thus almost all intrinsically luminous CT AGNs (unobscured $L_{\text {rest }-2-10 \mathrm{keV}}>10^{44} \mathrm{erg} \mathrm{s}^{-1}$ ) have detectable AGN mid-IR emission, consistent with recent IR studies of high-luminosity AGNs (Mateos et al. 2013). Figure 7 shows the fraction of CT AGNs with $\mathrm{EW}_{6.2 \mu \mathrm{m}}<0.4 \mu \mathrm{m}$ as a function of the $2-10 \mathrm{keV}$ flux. Above $10^{-15} \mathrm{erg} \mathrm{s}^{-1} \mathrm{~cm}^{-2}$ where intrinsic bright CT AGNs dominate, $80 \%-90 \%$ of CT AGNs have $\mathrm{EW}_{6.2 \mu \mathrm{m}}<0.4 \mu \mathrm{m}$. Below $10^{-15} \mathrm{erg} \mathrm{s}^{-1} \mathrm{~cm}^{-2}$, the fraction drops with decreasing flux but still remains appreciable, around $60 \%$ and $30 \%$ at $2-10 \mathrm{keV}$ flux of $10^{-16} \mathrm{erg} \mathrm{s}^{-1} \mathrm{~cm}^{-2}$ and $10^{-17} \mathrm{erg} \mathrm{s}^{-1} \mathrm{~cm}^{-2}$, respectively.

The reliable identification of CT AGNs beyond the local universe is still a challenge, even though multi-wavelength tools that combine the X-ray data with optical/IR photometry have been developed that reveal many candidates. The upper panel of Figure 8 shows the distribution of AGNs in the plane 


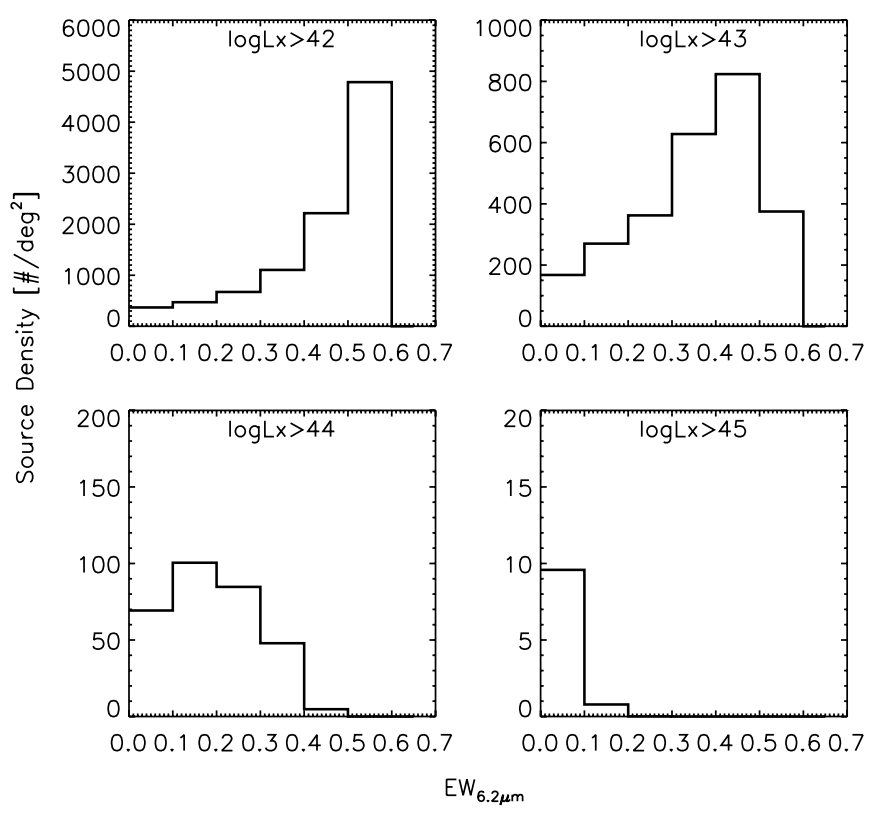

Figure 6. Predicted distribution of $6.2 \mu \mathrm{m}$ aromatic feature EW for CT AGNs above different intrinsic rest-frame $2-10 \mathrm{keV}$ luminosities. In our model, pure star formation has an EW of $0.6 \mu \mathrm{m}$; any value below that is due to contributions from AGN emission.

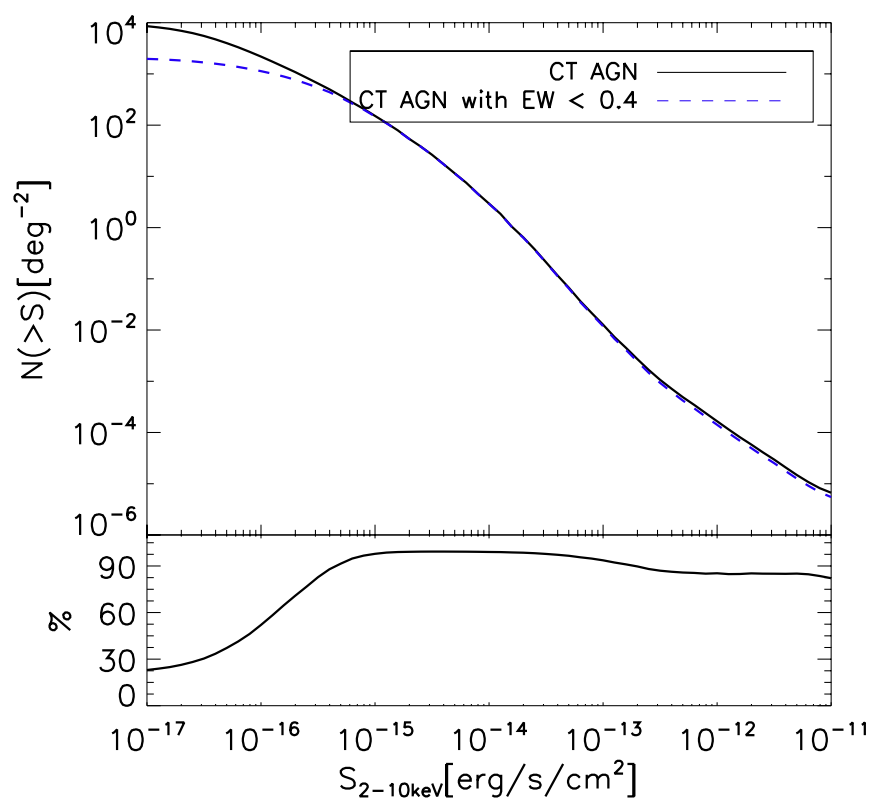

Figure 7. Cumulative surface density of CT AGNs (intrinsic rest-frame 2-10 keV luminosity above $10^{42} \mathrm{erg} \mathrm{s}^{-1}$ and $\mathrm{HI}$ column density above $10^{24} \mathrm{~cm}^{-2}$ ) and CT AGNs with $6.2 \mu \mathrm{m}$ aromatic feature EW lower than 0.4 (from which the AGN mid-IR featureless emission can be detected).

(A color version of this figure is available in the online journal.)

of $\log \left(f_{2-10 \mathrm{keV}} / f_{0.5-2 \mathrm{keV}}\right)$ versus $\log \left(f_{\mathrm{IRAC}} \mu \mathrm{m} / f_{0.5}-2 \mathrm{keV}\right)$. The median positions of AGNs with $\log \left(N_{\mathrm{HI}} / \mathrm{cm}^{-2}\right)=21.5,22.5$, 23.5, 24.5, and 25.5 are labeled. Heavily obscured AGNs $\left(N_{\mathrm{HI}}>10^{23} \mathrm{~cm}^{-2}\right)$ are predicted to be well separated from the unobscured and mildly obscured objects $\left(N_{\mathrm{H}_{\mathrm{I}}}<10^{23} \mathrm{~cm}^{-2}\right)$. Note that in our model, the range in the distribution of a given $N_{\mathrm{H}}$ is mainly caused by a variation in the redshift-dependent $\mathrm{K}$ correction and contamination by star formation in the IRAC $-8 \mu \mathrm{m}$ band. It does not incorporate the effect of variation in the AGN SED at X-ray and IR wavelengths. Also shown in Figure 8 are the locations of the CDF-S sources from Xue
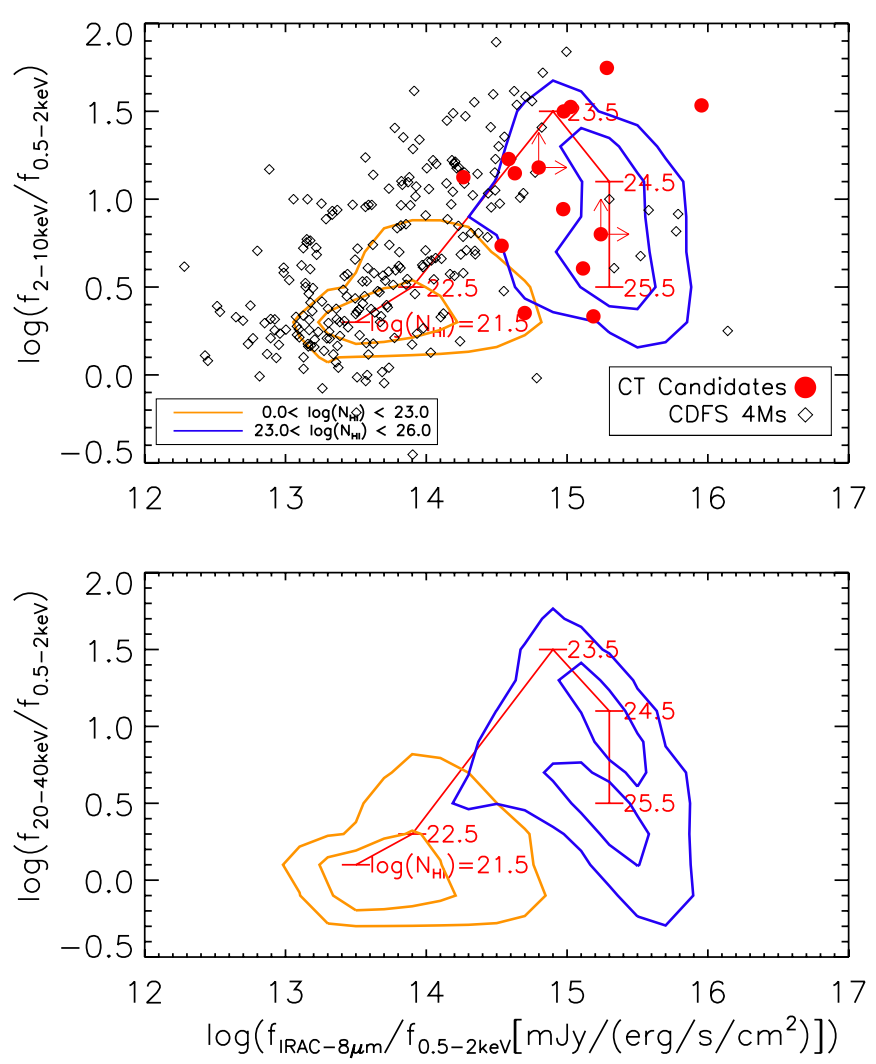

Figure 8. Distribution of AGNs with different $\mathrm{HI}_{\mathrm{I}}$ columns in the X-ray hardness vs. IR/X-ray ratio plane: $\log \left(f_{2}-10 \mathrm{kev} / f_{0.5-2 \mathrm{kev}}\right)$ vs. $\log \left(f_{\text {IRAC }}-8 \mu \mathrm{m} / f_{0.5-2 \mathrm{keV}}\right)$ for the upper panel and $\log \left(f_{20-40 \mathrm{keV}} / f_{0.5-2 \mathrm{keV}}\right)$ vs. $\log \left(f_{\mathrm{IRAC}-8 \mu \mathrm{m}} / f_{0.5-2 \mathrm{keV}}\right)$ for the lower panel. Orange and green contours are for unobscured and mildly obscured AGNs $\left(\log N_{\mathrm{H}_{\mathrm{I}}}<23\right)$ and heavily obscured AGNs $\left(\log N_{\mathrm{HI}_{\mathrm{J}}}>23\right)$, respectively. For each contour, two levels enclose $60 \%$ and $90 \%$ of objects, respectively. The diamond symbols give distributions of all CDF-S sources (Xue et al. 2011) while filled circles are CT AGN candidates from Alexander et al. $(2008,2011)$.

(A color version of this figure is available in the online journal.)

et al. (2011), which spread over a large region in this plane. However, the distributions of observed CT AGN candidates, as compiled from Alexander et al. $(2008,2011)$ lie roughly within our predicted range of heavily obscured AGNs, supporting the idea that heavily obscured AGNs can be identified through this simple diagnostic. The validity of a similar diagnostic plot has also been proposed by Severgnini et al. (2012) based on photometry of local confirmed CT AGNs. In the lower panel of Figure 8, we also examine the validity of $\log \left(f_{20-40 \mathrm{keV}} / f_{0.5-2 \mathrm{keV}}\right)$ versus $\log \left(f_{\text {IRAC } 8} \mu \mathrm{m} / f_{0.5-2 \mathrm{keV}}\right)$ in separating AGNs with different $\mathrm{H}_{\mathrm{I}}$ columns, which has a similar ability to the above diagnostic as shown in the upper panel.

Launched in 2012 June, NUSTAR (Harrison et al. 2013) should quickly help to constrain the distribution of CT AGNs. Figure 9 shows the predicted redshift distribution of all AGNs and CT AGNs above three flux limits targeted in NuSTAR surveys, namely $f_{10-30 \mathrm{keV}}>2 \times 10^{-14}, 4 \times 10^{-14}$, and $1.5 \times$ $10^{-13} \mathrm{erg} \mathrm{s}^{-1} \mathrm{~cm}^{-2}$. Given $0.3,1-2$, and $3 \mathrm{deg}^{2}$ for those three flux limits, respectively, our model predicts $\sim 100$ AGNs in total but only a few CT AGNs that will be detected by NuSTAR at 10-30 keV. Our predicted total number of AGNs is similar to recent predictions by Ballantyne et al. (2011), but our predicted number of CT AGNs is lower than theirs with the differences (a factor of 2-5) depending on the X-ray LFs they have adopted. 
The Astrophysical Journal, 777:6 (7pp), 2013 November 1
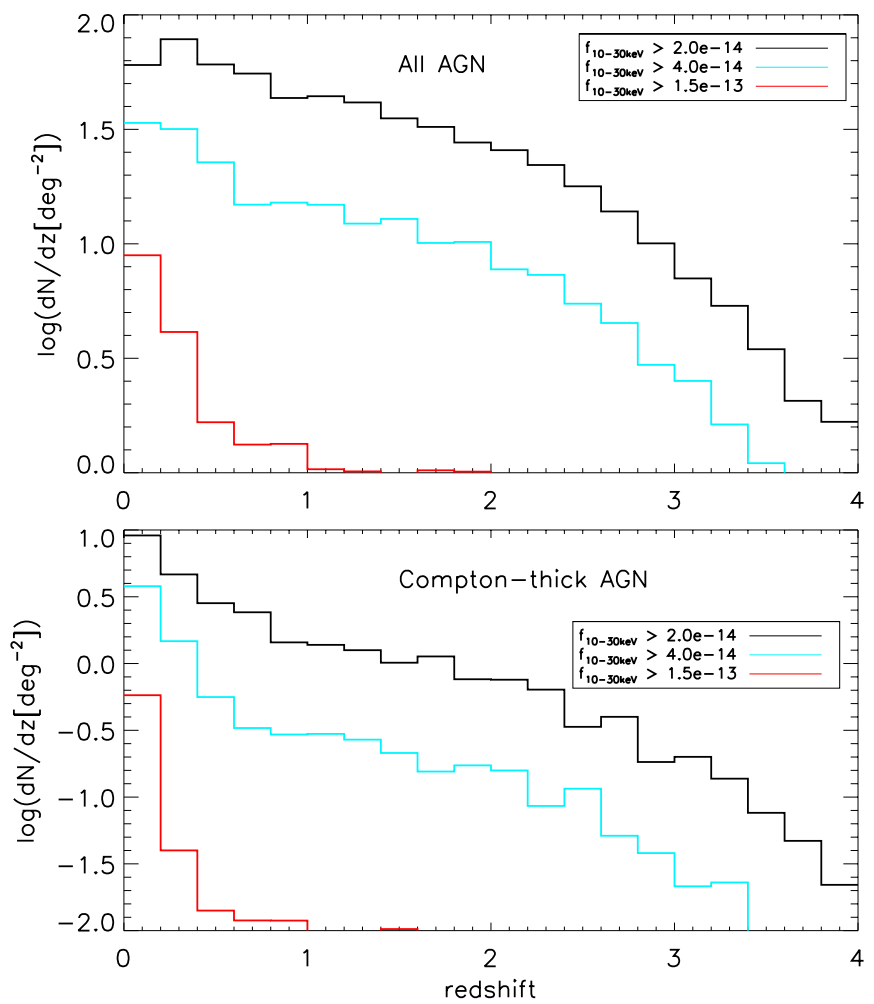

Figure 9. Predicted redshift distribution of NuSTAR AGNs (upper panel) and NuSTAR CT AGNs (lower panel) for three survey depths, as listed in Harrison et al. (2013).

(A color version of this figure is available in the online journal.)

\section{CONCLUSIONS}

We use our joint population model of X-ray and IR backgrounds to predict the CT AGN abundance and compare it to a diverse set of empirical determinations. The main conclusions are as follows.

1. At intrinsic $L_{\text {rest2-10 } \mathrm{keV}}>10^{42} \mathrm{erg} \mathrm{s}^{-1}$, the CT AGN density is predicted to be around a few $\times 10^{-4} \mathrm{Mpc}^{-3}$. The density of higher luminosity CT AGNs increases rapidly from $z=0$ to $z \sim 2-3$ and peaks at higher $z$.

2. The CT AGN fraction appears to be low $(2 \%-5 \%)$ at $f_{2-10 \mathrm{keV}}>10^{-15} \mathrm{erg} \mathrm{s}^{-1} \mathrm{~cm}^{-2}$ but increases rapidly at fainter flux levels.

3. The SMBH accretion in CT AGNs accounts for $38 \%$ of the total accreted SMBH mass and contributes to $25 \%$ of the CXB spectrum at its peak.

4. We also investigate the mid-IR spectra of CT AGNs based on techniques that have been developed to identify CT objects. The model predicts that the majority $(90 \%)$ of bright CT AGNs $\left(L_{\mathrm{rest} 2-10 \mathrm{keV}}>10^{44} \mathrm{erg} \mathrm{s}^{-1}\right.$ or $f_{2-10 \mathrm{keV}}>10^{-15} \mathrm{erg} \mathrm{s}^{-1} \mathrm{~cm}^{-2}$ ) have detectable hot dust emission from dusty tori; the fraction drops for faint objects, reaching $30 \%$ at $L_{\text {rest } 2-10 \mathrm{keV}}>10^{42} \mathrm{erg} \mathrm{s}^{-1}$ or $f_{2-10 \mathrm{keV}}>10^{-17} \mathrm{erg} \mathrm{s}^{-1} \mathrm{~cm}^{-2}$. Based on this, we conclude that heavily obscured AGNs $\left(N_{\mathrm{HI}_{\mathrm{I}}}>10^{23} \mathrm{~cm}^{-2}\right)$ can be separated from lower H I column AGNs through the plane of the observed-frame X-ray hardness versus mid-IR/X-ray ratio.
Shi, Helou, \& Armus

We thank the anonymous referee for helpful comments that have improved the paper significantly. The work is supported through the Spitzer 5MUSES Legacy Program 40539. The authors acknowledge support by NASA through awards issued by JPL/Caltech.

\section{REFERENCES}

Ajello, M., Alexander, D. M., Greiner, J., et al. 2012, ApJ, 749, 21 Ajello, M., Greiner, J., Sato, G., et al. 2008, ApJ, 689, 666 Alexander, D. M., Bauer, F. E., Brandt, W. N., et al. 2011, ApJ, 738, 44 Alexander, D. M., Chary, R.-R., Pope, A., et al. 2008, ApJ, 687, 835 Alonso-Herrero, A., Pérez-González, P. G., Alexander, D. M., et al. 2006, ApJ, 640, 167

Ballantyne, D. R., Draper, A. R., Madsen, K. K., Rigby, J. R., \& Treister, E. 2011, ApJ, 736, 56

Barger, A. J., Cowie, L. L., Mushotzky, R. F., et al. 2005, AJ, 129, 578 Brightman, M., \& Ueda, Y. 2012, MNRAS, 423, 702

Churazov, E., Sunyaev, R., Revnivtsev, M., et al. 2007, A\&A, 467, 529

Comastri, A., Setti, G., Zamorani, G., \& Hasinger, G. 1995, A\&A, 296, 1

Daddi, E., Alexander, D. M., Dickinson, M., et al. 2007, ApJ, 670, 173

Draper, A. R., \& Ballantyne, D. R. 2009, ApJ, 707, 778

Draper, A. R., \& Ballantyne, D. R. 2010, ApJL, 715, L99

Elmegreen, B. G., \& Burkert, A. 2010, ApJ, 712, 294

Fabian, A. C. 1999, MNRAS, 308, L39

Fiore, F., Puccetti, S., Brusa, M., et al. 2009, ApJ, 693, 447

Förster Schreiber, N. M., Genzel, R., Lehnert, M. D., et al. 2006, ApJ, 645, 1062

Gendreau, K. C., Mushotzky, R., Fabian, A. C., et al. 1995, PASJ, 47, L5

Genzel, R., Burkert, A., Bouché, N., et al. 2008, ApJ, 687, 59

Georgantopoulos, I., Akylas, A., Georgakakis, A., \& Rowan-Robinson, M. 2009, A\&A, 507, 747

Gilli, R., Comastri, A., \& Hasinger, G. 2007, A\&A, 463, 79

Gruber, D. E., Matteson, J. L., Peterson, L. E., \& Jung, G. V. 1999, ApJ, 520,124

Guainazzi, M., Matt, G., \& Perola, G. C. 2005, A\&A, 444, 119

Harrison, F. A., Craig, W. W., Christensen, F. E., et al. 2013, ApJ, 770, 103

Hasinger, G., Miyaji, T., \& Schmidt, M. 2005, A\&A, 441, 417

Hopkins, P. F., Hayward, C. C., Narayanan, D., \& Hernquist, L. 2012, MNRAS, 420,320

Hopkins, P. F., Richards, G. T., \& Hernquist, L. 2007, ApJ, 654, 731

Lacy, M., Storrie-Lombardi, L. J., Sajina, A., et al. 2004, ApJS, 154, 166

Law, D. R., Steidel, C. C., Erb, D. K., et al. 2007, ApJ, 669, 929

Luo, B., Brandt, W. N., Xue, Y. Q., et al. 2011, ApJ, 740, 37

Mainieri, V., Bergeron, J., Hasinger, G., et al. 2002, A\&A, 393, 425

Marconi, A., Risaliti, G., Gilli, R., et al. 2004, MNRAS, 351, 169

Mateos, S., Alonso-Herrero, A., Carrera, F. J., et al. 2013, arXiv:1305.7237

Moretti, A., Pagani, C., Cusumano, G., et al. 2009, A\&A, 493, 501

Perola, G. C., Puccetti, S., Fiore, F., et al. 2004, A\&A, 421, 491

Piccinotti, G., Mushotzky, R. F., Boldt, E. A., et al. 1982, ApJ, 253, 485

Piconcelli, E., Cappi, M., Bassani, L., Di Cocco, G., \& Dadina, M. 2003, A\&A, 412, 689

Polletta, M. d. C., Wilkes, B. J., Siana, B., et al. 2006, ApJ, 642, 673

Revnivtsev, M., Gilfanov, M., Sunyaev, R., Jahoda, K., \& Markwardt, C. 2003, A\&A, 411, 329

Risaliti, G., Maiolino, R., \& Salvati, M. 1999, ApJ, 522, 157

Severgnini, P., Caccianiga, A., \& Della Ceca, R. 2012, A\&A, 542, A46

Shankar, F., Salucci, P., Granato, G. L., De Zotti, G., \& Danese, L. 2004, MNRAS, 354, 1020

Shi, Y., Helou, G., Armus, L., Stierwalt, S., \& Dale, D. 2013, ApJ, 764, 28

Stern, D., Eisenhardt, P., Gorjian, V., et al. 2005, ApJ, 631, 163

Stierwalt, S., Armus, L., Surace, J. A., et al. 2013, ApJS, 206, 1

Tacconi, L. J., Genzel, R., Neri, R., et al. 2010, Natur, 463, 781

Tozzi, P., Gilli, R., Mainieri, V., et al. 2006, A\&A, 451, 457

Treister, E., Cardamone, C. N., Schawinski, K., et al. 2009a, ApJ, 706, 535

Treister, E., Natarajan, P., Sanders, D. B., et al. 2010, Sci, 328, 600

Treister, E., Urry, C. M., \& Virani, S. 2009b, ApJ, 696, 110

Türler, M., Chernyakova, M., Courvoisier, T. J.-L., et al. 2010, A\&A, 512, A49

Wada, K., \& Norman, C. A. 2002, ApJL, 566, L21

Wu, Y., Helou, G., Armus, L., et al. 2010, ApJ, 723, 895

Xue, Y. Q., Luo, B., Brandt, W. N., et al. 2011, ApJS, 195, 10

Yu, Q., \& Tremaine, S. 2002, MNRAS, 335, 965 